\title{
Effects of Exogenous Melatonin on Kiwifruit Seedlings Growth under Weak Light Treatment
}

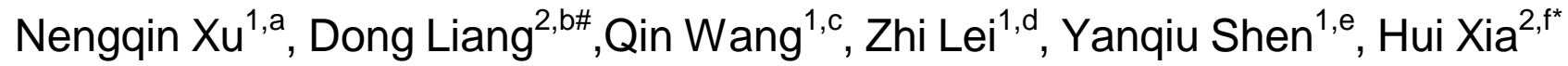 \\ ${ }^{1}$ College of Horticulture, Sichuan Agricultural University, Chengdu, Sichuan, China \\ ${ }^{2}$ Institute of Pomology and Olericulture, Sichuan Agricultural University, Chengdu, Sichuan, China \\ a1173604537@qq.com, ${ }^{\mathrm{b}} 756141969 @ q q$. com $^{\text {\# }}$, ${ }^{\mathrm{c}}$ 18428385472@163.com, ${ }^{\mathrm{d}}$ 1280195400@qq.com, \\ 517590097@qq.com, susanxia_2001@163.com * \\ ${ }^{\#}$ Co-first author, ${ }^{*}$ Corresponding author
}

Keywords: melatonin, kiwifruit, weak light treatment, physiological indexes

Abstract. To investigate the effect of exogenous melatonin (MT) on the growth of kiwifruit seedling under weak light treatment, in this study, kiwifruit seedling was used as the experimental material, applied $100 \mu \mathrm{mol} / \mathrm{L}$ MT solution by root irrigation, and determined physiological indexes under low light intensity. The results provide theoretical basis for improving the yield and quality of kiwifruit. The results showed that the chlorophyll and carotenoid content of kiwifruit leaves increased significantly under weak light treatment, but there was no significant relationship between the application of MT; The content of soluble sugar decreased firstly and then increased, the contents of soluble protein, CAT and POD increased substantially; Root irrigation MT solution can increased the degradation of soluble sugar and CAT, change the trend of soluble protein content and POD.

Kiwifruit was called as the 'King of VC', due to its rich nutrition, medicine and VC content. In recent years, agricultural facility has developed rapidly, but the facilities cover and frame structure have a bad effect on light conditions, and spring or autumn seasons often appear cloudy and rainy for several days, which cause plants grow in low light stress. Melatonin (MT) is a small molecule that is widely present in animals, prokaryotes and plants, it has been widely concerned since its discovery in the pineal gland of cattle since $1958^{[1]}$. MT has the ability to scavenge free radicals to protect cells from injury and improve the ability of plants to resist stress in plants, such as resistance to heavy metal stress , anti-aging, salt tolerant, disease resistant, drought resistant, anti UV capacity. In this experiment, one-year-old wild kiwifruit seedling was used as materials, processing from the aspects of photosynthetic pigment content, osmotic adjustment substances and antioxidant enzymes; the function of MT in delaying leaf senescence was analyzed from physiological level, which provided a theoretical basis for the rational use of MT to solve the problem of weak light stress.

\section{Material and Methods}

Plant materials and treatment. Seed of kiwifruit was collected in September, 2015. After stored at $4{ }^{\circ} \mathrm{C}$ for two months, seeds were treated with variable temperature at $4^{\circ} \mathrm{C} 16 \mathrm{~h}, 24^{\circ} \mathrm{C} 8 \mathrm{~h}$ for two weeks, and then, placed at $25^{\circ} \mathrm{C}$ for $10-15$ days to germinate. Sowed the sprouting seeds, and placed them in the artificial climate chamber (light-dark cycle: $12 \mathrm{~h} / 12 \mathrm{~h}$, diurnal temperature: $25^{\circ} \mathrm{C} / 20^{\circ} \mathrm{C}$ ). When had 2-3 true leaves, 45 potted uniform plants were selected and randomly divided into 3 groups, each group of 15 pots, and three plants in each pot. In the first 8 days before the start of the weak light treatment, Group 3 was fed with $100 \mu \mathrm{mol} / \mathrm{L}$ MT solution every two days, 20ml per pot; Group 1 and Group 2 were irrigated with equal volume of water. After 5 times of MT root irrigation, group 2 and group 3 were subjected to weak light treatment, the day was designed as 0 day, and weak light conditions for two sunshade nets covering treatment(60\%). The treatments were as follows: (1)CK: normal light, clear water treatment; (2) T1: weak light, clear water treatment; (3)T2: weak light, $100 \mu \mathrm{mol} / \mathrm{L}$ MT treatment. 6-9 leaves from randomly 3 pots were collected on the $0,2,4,8$ days, and frozen and stored in the $-80^{\circ} \mathrm{C}$ for physiological parameters measuring. 
Physiological indexes determination and Enzyme assays. Determination of chlorophyll content was referenced Arnon's method ${ }^{[2]}$ and improved. Soluble sugar content was determined using anthrone colorimetric method; content of soluble protein was determined by Kamas brilliant blue G-250 staining method, The activity of peroxidase (POD) was determined by methyl catechol colorimetric method, with the spectrophotometric determination of catalase (CAT) activity ${ }^{[3]}$. The above indexes were repeated for 9 times, and the average value was taken as the measured value.

Data analysis Using Excel 2010 for data processing and chart drawing, SPSS Statistics 22 for statistical analysis, data statistics using one-way ANOVA method of Duncan test for each variable ( $p$ $<0.05)$. Data were expressed as mean \pm standard deviation (SD).

\section{Results and Discussion}

Chlorophyll content and carotenoid content. Chlorophyll and carotenoids play an important role in photosynthesis ${ }^{[4]}$.Figure 1 shows that the photosynthetic pigment slightly decreased, may be the light is too strong in July, resulting in photosynthetic pigment decomposed ${ }^{[5]}$. The chlorophyll and carotenoid contents of T1 and T2 showed a slow growth trend under the weak light, on the 8th days, the chlorophyll and carotenoid contents of T1 were 1.23 times and 1.30 times of CK, and they were 1.30 and 1.35 times of $\mathrm{CK}$ in $\mathrm{T} 2$, These shows that kiwifruit as shade plants, a certain degree of shading a short time is beneficial to the increase of photosynthetic pigment. Liu ${ }^{[6]}$ was consistent with the results obtained of peach tree under weak light treatment, Huang ${ }^{[7]}$ has also got the results of chlorophyll content increased in the low light treatment. The possible reasons are as follows: first, moderate weak light reduced the decomposition of photosynthetic pigment. second, plants have the ability to actively adapt to the growth environment of the light conditions ${ }^{[8]}$, the kiwifruit seedlings adjust to the weak light stress by synthesizing more photosynthetic pigments.T2 compared with T1, chlorophyll and carotenoid synthesis increased, but not significant, in other words, the effect of MT on the photosynthetic pigment under weak light stress was not significant.
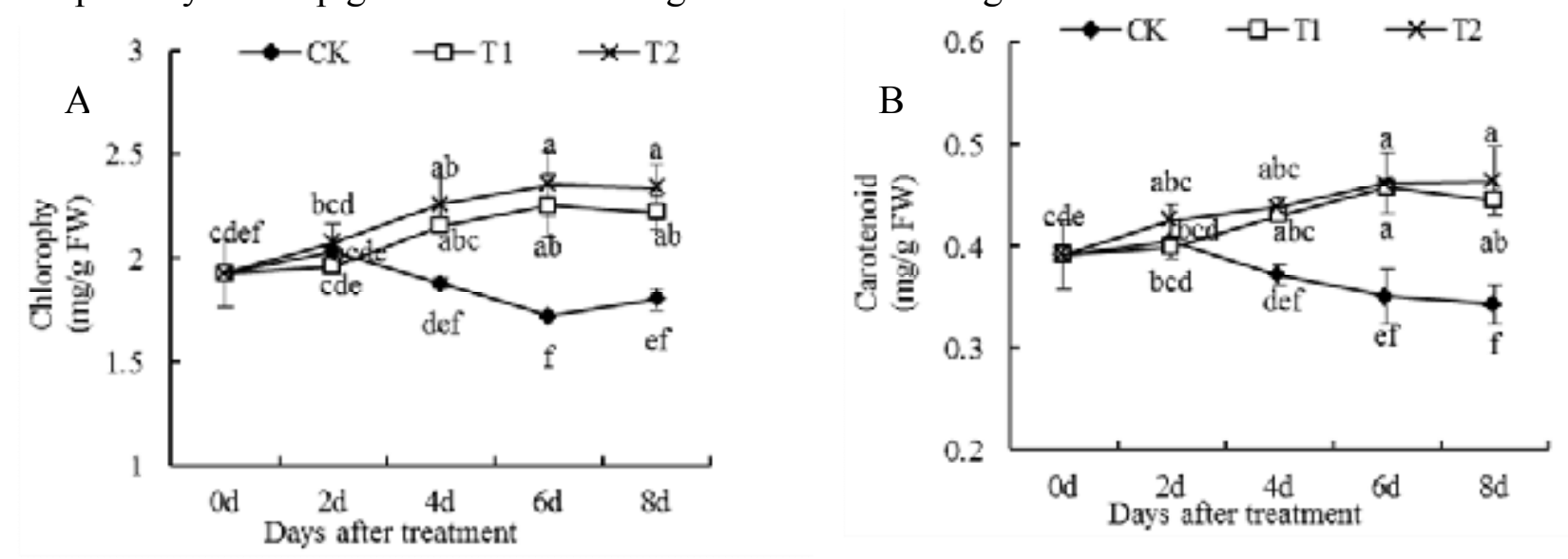

Fig. 1 Effects of exogenous melatonin on contents of Chlorophyll and Carotenoid of kiwifruit leaves

Soluble sugar and soluble protein. Soluble sugar is the main osmotic regulator of plants under stress, and it is also a way of energy storage under adverse circumstances ${ }^{[9]}$. Figure 2.A shows that, compared with $\mathrm{CK}$, the soluble sugar content of $\mathrm{T} 1$ and $\mathrm{T} 2$ is gradually reduced. $\mathrm{Xu}^{[10]}$ on cucumber to weak light treatment consistent with the results obtained, the reason is that the shading treatment led to the lack of illumination, the inhibition of photosynthesis and the decrease of sugar content in plants; On the 4th days, the soluble sugar began to recover slowly, indicating that the kiwifruit have the mechanism of active adaptation to weak light. Compared with T1, the soluble sugar of leaves in T2 was maintained at a relatively high level, indicating that MT has a role in increase photosynthesis in plants under low light environment.

The soluble protein in plants as another important osmotic adjustment, various adversities cause the change of protein quantity, it is possible that the normal soluble protein synthesis is blocked or the protein breaks down, resulting in the decrease of the content, and may also appear that a number of 
stress-related proteins are induced to increase the content of the protein ${ }^{[11,12]}$. Figure 2.B shows the experiment of $40 \%$ shading treatment increased the soluble protein content in kiwifruit leaves, indicating its synthesis is greater than the degradation rate, and more proteins associated with weak light stress were expressed; soluble protein content of the T2 tend to much smoother compared with $\mathrm{T} 1$, indicating exogenous melatonin may have a role in stabilizing soluble protein.
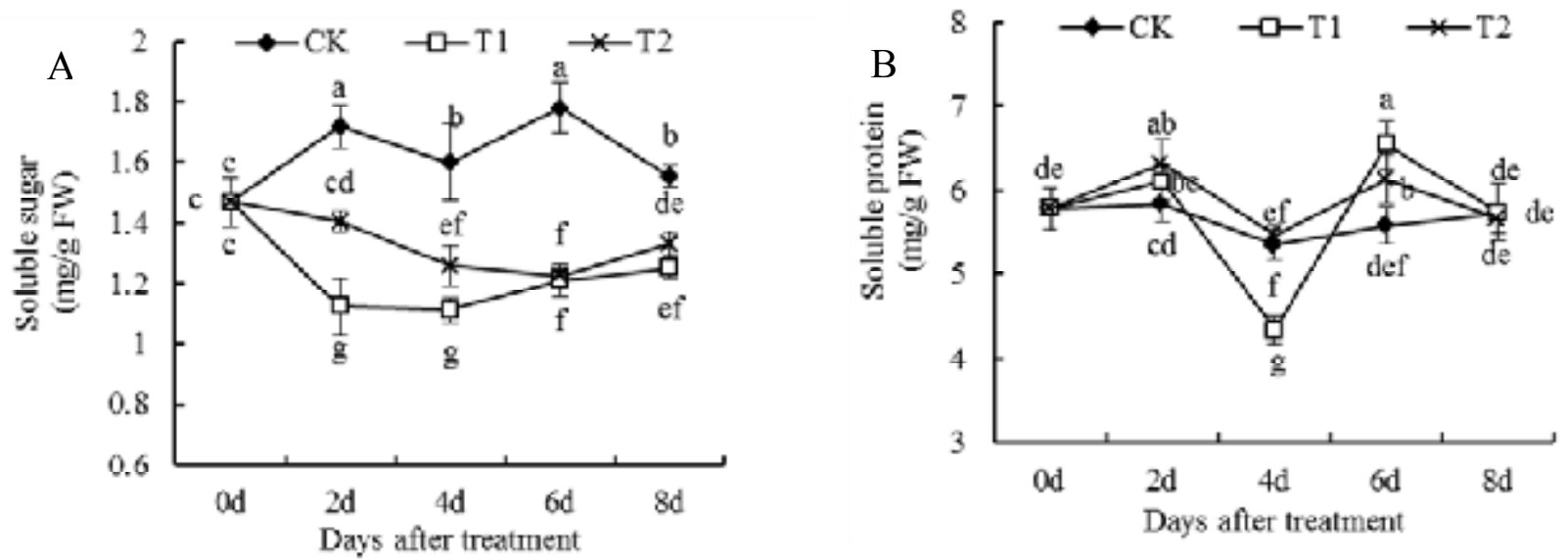

Fig.2 Effects of exogenous melatonin on contents of soluble sugar and soluble protein of kiwifruit leaves

CAT and POD activity. Catalase (CAT) and peroxidase (POD) are present in all plant cells, and are an important part of the active oxygen protective enzyme system, they can scavenge reactive oxygen species in the process of stress or aging, protect the membrane structure, so that the plants can resist adversity to a certain degree ${ }^{[13]}$. Figure 3 shows that CAT and POD contents of kiwifruit in leaves increased first and then decreased in weak light stress, the first increase in enzyme activity may be due to the rapid accumulation of reactive oxygen species, which results in the synthesis of protective enzymes, this is a stress response of kiwifruit seedlings to weak light; the decrease may be due to the prolongation of the weak light stress, the excess reactive oxygen species can't be completely removed, resulting in reduced enzyme activity. On the second days, two kinds of enzyme activity of T1 was higher than T2, which indicates that MT pretreatment had a delayed effect on the change of protective enzymes, that shows MT as an antioxidant to remove some of the reactive oxygen species, so that the accumulation of reactive oxygen species is not significant in T1, thus delaying the induction of protective enzymes. In addition, CAT reached the maximum on fourth days, T1 and T2 were 2.31 and 2.76 times of $\mathrm{CK}$, respectively; POD reached the maximum on sixth days, T1 and T2 were 3.59 and 3.22 times of CK. This shows the response time and the expression level of CAT and POD protective enzymes were different, and the effect of MT on the regulation of the expression of protective enzymes was selective.
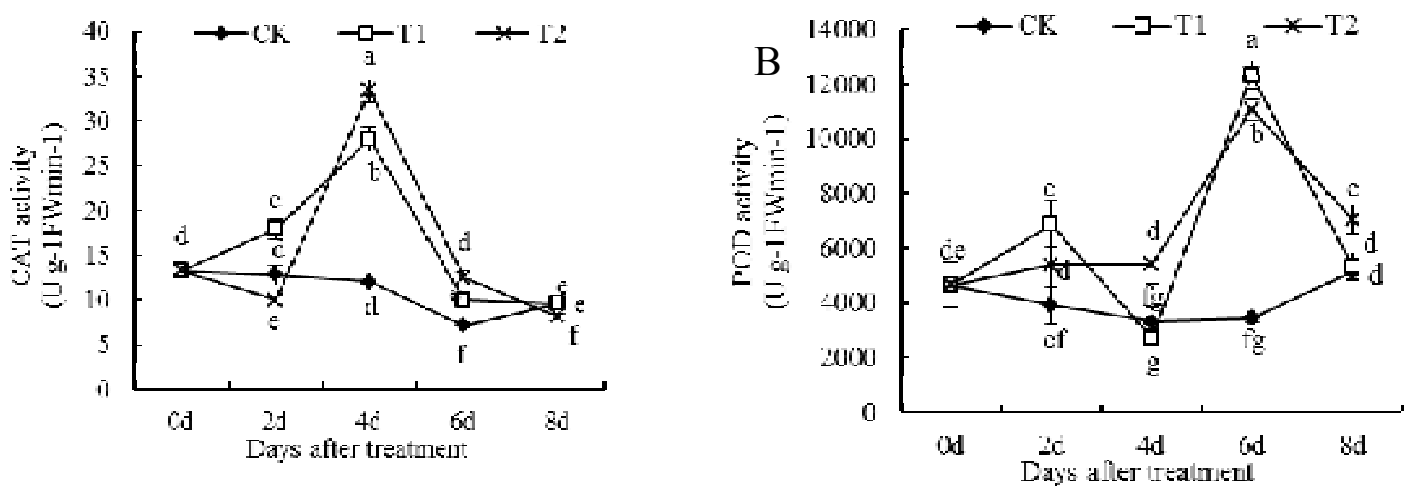

Fig.3 Effects of exogenous melatonin on contents of CAT activity and POD activity of kiwifruit leaves

\section{Conclusions}

In conclusion, plants have the ability to actively adapt to the growth environment. Moderate shading treatment reduced the soluble sugar content of kiwifruit, decreased the intensity of light damage, 
induced the increase of photosynthetic pigment and antioxidant enzyme activity, it is beneficial to the growth and development of kiwifruit in a certain extent. Melatonin for promoting the effect of photosynthetic pigment synthesis is not significant, but can increase the content of soluble sugar, the regulation of protective enzyme activity, but also has the function of stabilizing soluble protein, thereby reducing the weak light stress of kiwifruit plants.

\section{Acknowledgements}

This work was financially supported by the Sichuan Technical Foundation (02016NZ0105).

\section{References}

[1] H. Rudlger: Melatonin and the theories of aging: a critical appraisal of melatonin's role in anti-aging mechanisms. J. Pineal Res, 55:325-365(2013).

[2] M. Ma, C. Ma and Z. Xiao: Study on Extraction of chlorophyll from kiwifruit leaves. food industry science and technology (2006).

[3] X. Wang and J. Huang: Principle and technology of plant physiology and biochemistry experiment. Beijing: Higher Education Press (2015).

[4] X.L. Sun, Y.F. Xu and L.X. Ma: Leaf photosynthetic pigment formation in response to shading. Journal of plant ecology 34 (8): 989 - 999(2010).

[5] Z. Wang: Plant Physiology (China Agriculture Press, Beijing 2000)

[6] W.H. Liu and D. Gao: Effects of different light intensity characteristics of photosynthesis and chlorophyll fluorescence of peach tree. China agricultural science, 39(10): 2069-2075(2006).

[7] W.D. Huang, L.K. Wu and J.C. Zhan: Growth and Photosynthesis Adaptation of Dwarf-Type Chinese Cherry (Prunus pseudocerasus L. cv. Laiyang) Leaves to Weak Light Stress. Scientia Agricultura Sinica. 37(12):1981-1985 (2004).

[8] J.C. Zhan, W.D. Huang, and L.K. Wu: Research of Weak Light Stress Physiology in Plants. Chinese Bulletin of Botany. 20(1):43-50(2003)

[9] Z.Q. Liu and S.C. Zhang: Plant Resistance Physiology (China Agriculture Press, Beijing 1994)

[10] X.C. Xu: Soluble sugar contents in leaves and stems of greenhouse cucumber exposed to weak light. Jiangsu J. of Agr. Sci,31(6):1448-1450(2015).

[11] W. Huang, L. Wang and Kyrgyzstan: Weak light stress on plant physiology. Chinese Bulletin of Botany, 20 (1): 43-50(2003)

[12] H.X. Xu, X. Zhang and S.M. Wang: Genetic Diversity of Achnatherum Splendens. Journal of Anhui Agricultural Sciences, 29(1): 21-23(2008).

[13] J.X Zhang and M.B. Kirkham: Drought-stress-induced changes in activities of superoxide dismutase,catalase, and peroxidase in wheat species. Plant Cell Physiol. 35(5):785-791(1994). 